\title{
Molecular pathology of Sandhoff disease with p.Arg505GIn in HEXB: application of simulation analysis
}

\author{
Naoko Yasui ${ }^{1,5}$, Yutaka Takaoka ${ }^{2,5}$, Hisahide Nishio ${ }^{3,5}$, Dian K Nurputra ${ }^{3}$, Kenji Sekiguchi ${ }^{1}$, \\ Hirotoshi Hamaguchi ${ }^{1}$, Hisatomo Kowa ${ }^{1}$, Eiichi Maeda ${ }^{2}$, Aki Sugano ${ }^{2}$, Kenji Miura ${ }^{2}$, Toshiyuki Sakaeda ${ }^{4}$, \\ Fumio Kanda ${ }^{1}$ and Tatsushi Toda ${ }^{1}$
}

Sandhoff disease is a GM2 gangliosidosis caused by mutations in HEXB encoding the $\beta$-subunit of $\beta$-hexosaminidase $A$. $\beta$-Hexosaminidase $A$ exists as a heterodimer consisting of $\alpha$ - and $\beta$-subunits, and requires a GM2 activator protein to hydrolyze GM2. To investigate the molecular pathology in an adult Sandhoff disease patient with an early disease onset, we performed mutation detection, western blot analysis and molecular simulation analysis. The patient had compound heterozygous mutations p.Arg505GIn and p.Ser341ValfsX30. Western blot analysis showed that the amount of mature form of the $\alpha$ - and $\beta$-subunits was markedly decreased in the patient. We then performed docking simulation analysis of the $\alpha$ - and $\beta$-subunits with p.Arg505GIn, the GM2AP/GM2 complex and $\beta$-hexosaminidase A, and GM2 and $\beta$-hexosaminidase A. Simulation analysis showed that p.Arg505GIn impaired each step of molecular conformation of the $\alpha$ - and $\beta$-subunits heterodimer, the activator protein and GM2. The results indicated that p.Ser341ValfsX30 reduced the amount of $\beta$-subunit, and that $p$.Arg505GIn hampered the maturation of $\alpha$ - and $\beta$-subunits, and hindered the catalytic ability of $\beta$-hexosaminidase $A$. In conclusion, various methods including simulation analysis were useful to understand the molecular pathology in Sandhoff disease. Journal of Human Genetics (2013) 58, 611-617; doi:10.1038/jhg.2013.68; published online 13 June 2013

Keywords: $\beta$-hexosaminidase; GM2 gangliosidosis; HEXB; molecular simulation analysis; p.Arg505GIn; p.Ser341ValfsX30; Sandhoff disease

\section{INTRODUCTION}

Sandhoff disease (SD) is an autosomal recessive disorder resulting in accumulation of the ganglioside GM2 in the nervous system because of mutations in HEXB. ${ }^{1}$ The prevalence of Sandhoff disease in nonJewish populations is 1 in $\sim 310000$ live births. Sandhoff carrier frequency in non-Jewish populations is 1 in $\sim 278 .{ }^{1}$ More than 20 different mutations in HEXB are reported. ${ }^{2}$

SD is classified into three phenotypes: infantile SD; juvenile SD; and adult SD. ${ }^{2}$ Infantile SD and juvenile SD usually follow an acute or subacute clinical course, and patients die 3-10 years after disease onset. Young patients demonstrate widespread symptoms related to the nervous system, including motor weakness, hypotonia, mental retardation, dysarthria, visual disturbance, seizure, ataxia, diarrhea and constipation. ${ }^{2,3}$ In contrast, adult SD patients display a chronic disease course with the nervous system affected by spinocerebellar degeneration, ${ }^{4-7}$ motor neuron disease, ${ }^{7-10}$ and sensory or autonomic dominant peripheral neuropathy. ${ }^{11}$
SD results from mutations in $H E X B$ encoding the $\beta$-subunit that is shared between $\beta$-hexosaminidase ( $\beta$-Hex) A and $\beta$-Hex B. $\beta$-Hex A exists as a heterodimer consisting of $\alpha$ - and $\beta$-subunits ( $\alpha \beta$-heterodimer), whereas $\beta$-Hex B exists as a homodimer consisting of the $\beta$-subunit $\left(\beta \beta\right.$-homodimer). ${ }^{2} \quad$ Enzymatic analysis with 4-methylumbelliferyl (4-MU) substrates showed reduced catalytic abilities of $\beta$-Hex $A$ and $\beta$-Hex $B$ in SD. $^{2,12,13}$ The decreased catalytic abilities of the enzymes with 4-MU substrates can be explained by the presence of the mutant $\beta$-subunit shared between $\beta$-Hex $A$ and $\beta$-Hex B. ${ }^{13}$ Hydrolysis of 4 -MU substrates does not require the presence of the GM2 activator protein (GM2AP), but the presence of GM2AP is essential for hydrolysis of GM2 in humans. ${ }^{2,14}$ Enzymatic analysis with 4-MU substrates cannot therefore fully reflect the in vivo functions of $\beta-\mathrm{Hex} A$ and $\beta$-Hex $B$.

Reduced activity of mutant $\beta$-Hex A may be the primary cause of accumulation of GM2 in SD, as GM2AP specifically binds to $\beta$-Hex A. ${ }^{2,14}$ However, it is not clear if mutant $\beta$-Hex B contributes to the

\footnotetext{
${ }^{1}$ Division of Neurology, Kobe University Graduate School of Medicine, Kobe, Japan; ²Division of Medical Informatics and Bioinformatics, Kobe University Hospital, Kobe, Japan; ${ }^{3}$ Division of Epidemiology, Kobe University Graduate School of Medicine, Kobe, Japan and ${ }^{4}$ Center for Development of Integrative Education in Pharmacy and Pharmaceutical Sciences, Graduate School of Pharmaceutical Sciences, Kyoto University, Kyoto, Japan

Correspondence: Dr N Yasui, Division of Neurology, Kobe University Hospital, 7-5-2, Kusunoki-cho, Chuo-ku, Kobe 650-0017, Japan.

E-mail: nyasui@med.kobe-u.ac.jp

${ }^{5}$ These authors contributed equally to this work.

Received 1 November 2012; revised 15 May 2013; accepted 19 May 2013; published online 13 June 2013
} 
development of SD. Therefore, study of the pathogenesis of SD first requires examination of $\alpha \beta$-heterodimer formation and the catalytic activity of $\beta$-Hex A. The formation of the $\alpha \beta$-heterodimer in $\beta$-Hex A is critical not only for acquisition of catalytic ability but also for enzyme transport to lysosomes. ${ }^{15,16}$ Impairment of enzyme transport may also affect the residual activities of $\beta$-Hex A. Formation of the $\alpha \beta$-heterodimer in $\beta$-Hex $A$ is therefore important in GM2 metabolism because it may determine (1) the catalytic ability and lysosomal transport of $\beta$-Hex; (2) the residual activity of $\beta$-Hex A; and (3) the clinical phenotype of SD.

In this study, to clarify the molecular pathology of SD in an adult patient with a relatively early disease onset, we searched for HEXB mutations and performed molecular simulation analysis with the mutant $\beta$-subunit. Using molecular simulation, we analyzed the conformational stability of $\beta$-Hex A, binding of the GM2AP/GM2 complex to $\beta$-Hex A and GM2 hydrolysis. Insights into the molecular pathology of SD may lead to the development of novel treatments for lysosomal disorders.

\section{PATIENT AND METHODS}

\section{Patient}

The patient was a Japanese female born to healthy and non-consanguineous parents. At age 6, she had difficulty in running; at age 13, she could no longer run. Gait disturbance appeared and gradually worsened during high school. A neurological examination at age 20 revealed a prominent gait disturbance, ataxia in the limbs and trunk, and proximal muscle weakness and atrophy predominantly affecting the lower limbs. A cherry-red spot was absent in the macula of both eyes. Patellar tendon reflexes were weak. Pathological reflexes were absent. The intelligence quotient score was 71 on the Wechsler Adult Intelligence Scale-Revised. Brain magnetic resonance imaging revealed marked cerebellar atrophy, but $\mathrm{T}_{2}$-weighted images showed no abnormal intensity in the basal ganglia, thalamus and cerebral white matter (Figure 1). Nerve conduction studies were normal. Needle electromyography revealed chronic neurogenic patterns in the proximal muscles of the lower limbs.

The patient and her parents gave their informed consent for the enzyme activity assay and genetic analysis.

\section{Sample preparation}

Peripheral leukocytes were extracted using the following methods. Blood was collected using EDTA-coated tubes. To lyse erythrocytes, $28 \mathrm{ml}$ of ammonium chloride solution $\left(0.155 \mathrm{~mm} \mathrm{NH}_{4} \mathrm{Cl}, 10.0 \mathrm{~mm} \mathrm{KHCO}_{3}, 0.100 \mathrm{~mm}\right.$ EDTA2Na) was added to $7 \mathrm{ml}$ of blood and mixed. The mixture was centrifuged at $4{ }^{\circ} \mathrm{C}$ for $10 \mathrm{~min}$ at 1500 r.p.m., and the supernatant was removed. To wash the sediment, the following procedure was repeated twice: $15 \mathrm{ml}$ of normal saline solution was added to the mixture. The mixture was centrifuged at $4{ }^{\circ} \mathrm{C}$ for $10 \mathrm{~min}$ at 1500 r.p.m., and the supernatant was removed.

Genomic DNA was extracted from peripheral blood using a DNA extraction kit (SepaGene; Sanko Junyaku, Tokyo, Japan), according to the manufacturer's instructions.

Total protein was purified from peripheral leukocytes using Qiagen kits (QIAshredder, AllPrep kit; Qiagen, Venlo, The Netherlands), according to the manufacturer's instructions.

\section{Enzyme activity assay}

Enzyme activity was measured in the laboratory of SRL Inc (Tokyo, Japan) using the following methods. Peripheral leukocytes were prepared (as above). Leukocyte cell membranes were disrupted by sonication, and the supernatant after centrifugation was used for enzyme activity assay. To study $\beta$-Hex A activity, an aqueous reaction mixture $(100 \mu \mathrm{l})$ containing $50 \mu \mathrm{l}$ of cell extract, $6 \mathrm{~mm}$ 4-methylumbelliferyl- $N$-acetyl- $\beta$-D-glucosaminide-6-sulfate (4-MUGS), ${ }^{13}$ $0.1 \mathrm{M}$ citrate and $0.2 \mathrm{M}$ phosphate buffer $(\mathrm{pH} 4.0)$ was used. The reaction proceeded with incubation at $37^{\circ} \mathrm{C}$ and was stopped after $60 \mathrm{~min}$ by adding $1500 \mu \mathrm{l}$ of $0.2 \mathrm{M}$ glycine buffer ( $\mathrm{pH}$ 10.7). To study total $\beta$-Hex activity, a reaction mixture $(100 \mu \mathrm{l})$ containing $50.0 \mu \mathrm{l}$ of cell extract, $2 \mathrm{~mm}$ of 4 methylumbelliferyl- $N$-acetyl- $\beta$-D-glucosaminide $(4-\mathrm{MUG})^{13}$ and $0.1 \mathrm{M}$ citrate buffer ( $\mathrm{pH}$ 4.5) was used. The reaction proceeded with incubation at $37^{\circ} \mathrm{C}$ and was stopped after $30 \mathrm{~min}$ by adding $1500 \mu \mathrm{l}$ of $0.2 \mathrm{M}$ glycine buffer ( $\mathrm{pH} 10.7$ ). The activity of each enzyme was determined as the amount of the fluorescent molecule (4-MU) that emits light at $450 \mathrm{~nm}$ when excited by $365 \mathrm{~nm}$ light. Enzyme activity was adjusted according to the protein concentrations as measured using the Lowry method. ${ }^{17}$ Control studies of enzyme activity were performed using the enzyme obtained from healthy volunteers ( $\beta$-Hex A: $n=48$; total $\beta$-Hex: $n=100$ ) at SRL Inc.

\section{Genetic analysis of HEXB}

Polymerase chain reaction (PCR) amplification of each exon of HEXB in genomic DNA was performed using a PC-701 thermal cycler (Astec, Tokyo, Japan), according to the method of Narkis et al. ${ }^{18}$ The PCR fragment was then sequenced directly: the fragment underwent a cycle sequencing reaction using a BigDye Terminator v3.0 cycle sequencing kit (Applied Biosystems, Foster City, CA, USA), was applied to the genetic analyzer ABI PRISM 310 (Applied Biosystems) and was analyzed using DNA sequencing analysis software (Applied Biosystems).

\section{Western blot analysis}

The amounts of $\alpha$ - and $\beta$-subunits in peripheral leukocytes were determined by western blot analysis using rabbit anti- $\alpha$ - and $-\beta$-subunit antibodies (product code: ab91624 and ab140649, abcam, Cambridge, UK). The precursors of $\alpha$ - and $\beta$-subunits undergo post-translational proteolytic and glycosidic processing in the endoplasmic reticulum, Golgi and lysosomes to produce the mature form. ${ }^{2,19}$ Both antibodies also recognize these precursors of the subunits as well as the mature form. Mouse anti-tubulin antibodies were used as the basis for correction. Proteins were extracted as above and then subjected to sodium dodecylsulfate-polyacrylamide gel electrophoresis using a Mini-PROTEAN system and $4-20 \%$ precast polyacrylamide gel (Bio-Rad, Hercules, CA, USA). Proteins were then transferred to $0.45 \mu \mathrm{m}$ of nitrocellulose membrane using a Criterion Blotter (Bio-Rad), and visualized by immunostaining using the same antibodies as above and an ImageQuant LAS 4000 system (GE Healthcare UK Ltd., Buckinghamshire, UK).

\section{Simulation analysis}

Construction of the three-dimensional structure of $\beta$-Hex $A$. The known crystal structure of $\beta$-Hex A was the only mature form ${ }^{20}$ (Figure 2 ). Therefore, in this paper, based on the data of Lemieux et al. ${ }^{20}$ we performed simulation analysis of the mature forms of the $\alpha$-subunit, $\beta$-subunit and $\beta$-Hex A at those locations in lysosomes. ${ }^{2,20}$ The crystal structure of the $\beta$-Hex A octamer was

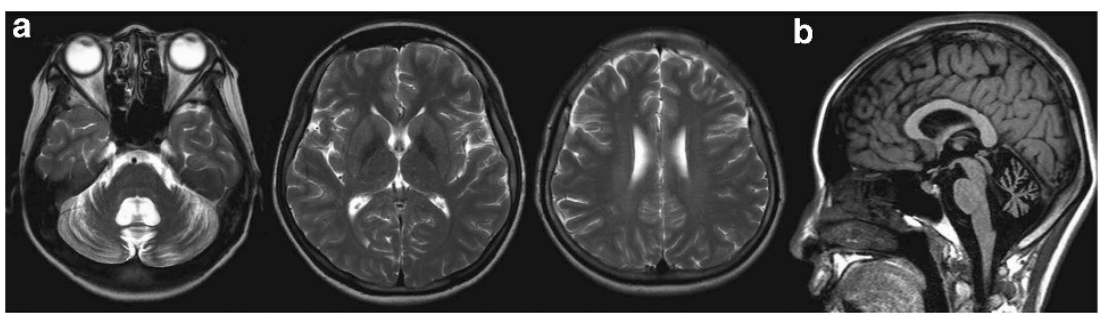

Figure 1 Brain MRI of the patient. (a) Three axial sections of T2-weighted images show marked cerebellar atrophy, but no abnormal intensity in the basal ganglia, thalamus and white matter. (b) Midline sagittal T1-weighted images show marked atrophy in cerebellar vermis. 


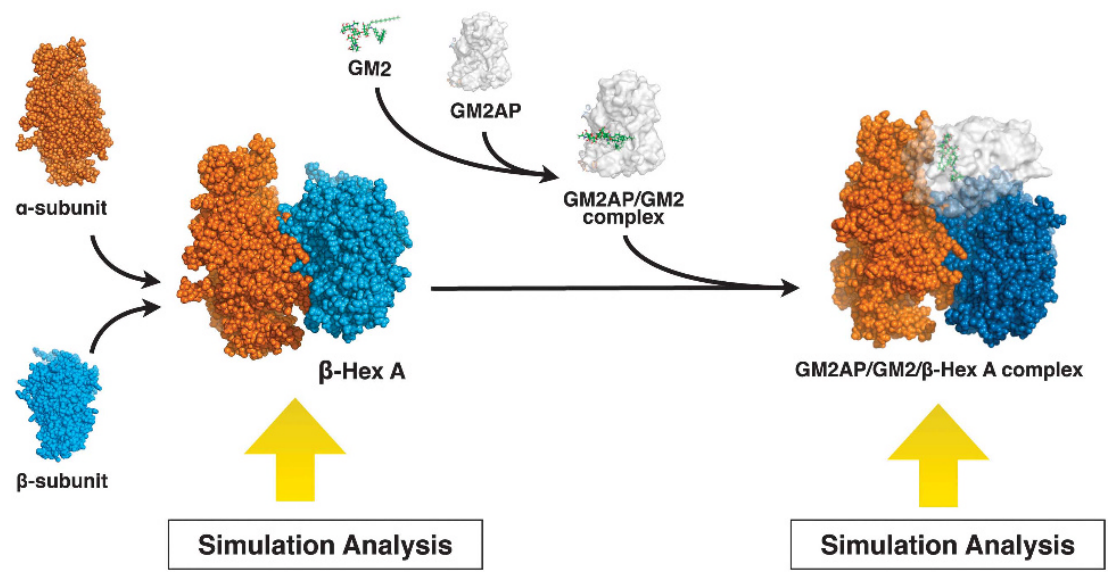

Figure 2 Schematic drawing of the steps in the structural formation of the GM2AP/GM2/ $\beta$-Hex A complex. In this process, we analyzed the conformational stability of the $\alpha \beta$-heterodimer (left simulation analysis) and the catalytic ability of $\beta$-Hex A (right simulation analysis).

downloaded from the Protein Data Bank (PDB; 2GJX), ${ }^{20}$ and a threedimensional (3-D) structure of the functional unit of the $\alpha \beta$-heterodimer (chain $\mathrm{A}$ and $\mathrm{B}$ ) ${ }^{19-21}$ was selected and subjected to energy minimization with MOE software (Molecular Operating Environment Version 2009.10, Chemical Computing Group Inc., Montreal, Quebec, Canada), which was used for homology modeling in a previous report. ${ }^{22}$ The 3-D structure of mutant $\beta$-Hex A was constructed by introducing the p.Arg505Gln mutation into the $\beta$-subunit and then performing energy minimization. Protein structures were protonated at $\mathrm{pH} 5.0$, that is, the same $\mathrm{pH}$ as that within lysosomes. The ionized residues in $\beta$-Hex A at pH 5.0 are listed in the Supplementary Information. The structures were soaked into water molecules and subjected to molecular mechanics calculations using the AMBER99 force field until the root mean square (RMS) gradient was less than $0.01 \mathrm{kcal} \mathrm{mol}^{-1} \AA^{-1}$. Following this, 100-ps molecular dynamics simulations were performed with a time step of $1.5 \mathrm{fs}$ at $300 \mathrm{~K}$ using the NVT (constant composition, volume and temperature) ensemble. The 3-D structure of $\beta$-Hex A obtained in this process was superimposed onto the structure of the $\alpha \beta$-heterodimer in PDB;2GJX (chain A and B). Then the root mean square deviation (RMSD) value of $1.01 \AA$ was calculated for all $\mathrm{C} \alpha$ atoms.

Docking simulation of $\alpha$ - and $\beta$-subunits, and conformational stability of $\beta$-Hex A. Docking simulation of the $\alpha$ - and $\beta$-subunits of $\beta$-Hex A was carried out to analyze the conformational stability of $\beta$-Hex A. In this analysis, we used ZDOCK software, as used by Wang et al. ${ }^{23}$ to evaluate the binding between molecules. Two thousand dockings of the $\alpha$ - and $\beta$-subunits were performed using ZDOCK with default parameters, and ZDOCK scores were analyzed. ${ }^{24}$ The docking site of each dimer was defined according to the crystal structure of $\beta$-Hex $A$, as described by Leimeux et al..$^{20}$

Construction of the 3-D structure of GM2AP/GM2 complex. The 3-D structure of the activator protein GM2AP was downloaded from the Protein Data Bank (PDBID: 1PU5). The 3-D structures of nine GM2 molecules were obtained from ChemSpider (ID: 1674492-5, 16744927-32). In this part of the analysis, we used MOE software with reference to past reports evaluating the substrate binding sites of enzymes. ${ }^{25} \mathrm{MOE}$ software was used for a total of 2000 docking runs of GM2AP and GM2, using the docking site described by Wright et al. ${ }^{26}$ The GM2AP/GM2 complex structures closest to the one described by Wright et al. ${ }^{26}$ were selected, and subjected to energy minimization to allow construction of the 3-D structure of the complex of GM2AP/GM2 and $\beta$-Hex A.

Docking simulation of GM2AP/GM2 complex and $\beta-H e x$ A. Five thousand docking runs of each of the nine GM2AP/GM2 complexes and $\beta$-Hex A were carried out six times using ZDOCK (total $45000 \times$ six runs). Docking sites of the GM2AP-GM2 complex and $\beta$-Hex A were defined according to a predicted model of the human Hex A-GM2-activator quaternary complex as reported by Mark et al..$^{19}$

Construction of the model structure of $\beta$-Hex A via GM2AP/GM2 complex. The 3-D GM2AP/GM2/ $\beta$-Hex A complex structures closest to the one predicted by Mark et al. ${ }^{19}$ were selected and subjected to energy minimization. GM2AP and GM2 were then removed from each complex. Energy minimization was performed before each removal step. The resultant structure of $\beta$-Hex A was used for the following simulation analysis.

Prediction of GM2 hydrolysis ability of $\beta$-Hex A. The GM2 hydrolysis ability of $\beta$-Hex A was predicted by means of docking simulations of GM2 and $\beta-\mathrm{Hex} A$, constructed as above. The docking site was defined at around Asp322 and Glu323 in the $\alpha$-subunit of $\beta$-Hex A, which, according to Lemieux et al. ${ }^{20}$ and Mark et al., ${ }^{19}$ is involved in ligand binding and/or protonation of the departing carbohydrate. For each of the nine $\beta$-Hex A, 5000 docking runs with GM2, which were used for construction of each $\beta$-Hex A, were performed using MOE DOCK. The docking experiments were performed six times (total $45000 \times$ six runs).

Statistical analysis. Statistical analysis was performed using R software. ${ }^{27}$ Differences in $S$ scores were evaluated using an unpaired Student's $t$ test. Data represent the mean \pm standard deviation (s.d.), with $P<0.05$ regarded as statistically significant.

\section{RESULTS}

\section{Enzyme activities}

$\beta$-Hex A and total $\beta$-Hex activities in the patient were 80.1 (19\% of the mean activity of the controls) and $88.6 \mathrm{nmol}$ per $\mathrm{mg}$ protein per hour $(10 \%$ of the mean activity of the controls), respectively (Table 1). Based on these lower $\beta$-Hex A and total $\beta$-Hex activities, the patient was diagnosed as having SD.

$\beta$-Hex A and total $\beta$-Hex activities of the mother were $284.8(66 \%$ of the mean activity of the controls) and $650.3 \mathrm{nmol}$ per $\mathrm{mg}$ protein per hour $(71 \%$ of the mean activity of the controls), respectively (Table 1).

$\beta$-Hex A and total $\beta$-Hex activities of the father were $428.8(100 \%$ of the mean activity of the controls) and $1146.2 \mathrm{nmol}$ per mg protein per hour (125\% of the mean activity of the controls), respectively (Table 1).

The difference in enzyme activity between the patient and her parents suggests that each of the parents possessed a different mutation. 
Table 1 Determination of $\beta$-Hex $A$ and total $\beta$-Hex activity in peripheral leukocytes with artificial substrates

\begin{tabular}{lcc}
\hline & $\begin{array}{c}\beta \text {-HexA (nmol per mg protein } \\
\text { per hour) }\end{array}$ & $\begin{array}{c}\beta \text {-Hex (nmol per mg protein } \\
\text { per hour) }\end{array}$ \\
\hline Patient & 80.1 & 88.6 \\
Mother & 284.8 & 650.3 \\
Father & 428.8 & 1146.2 \\
Controls & $429.3 \pm 89.1(n=48)$ & $913.9 \pm 256.1(n=100)$ \\
(mean \pm s.d.) & & \\
\hline
\end{tabular}

\section{Genetic analysis}

To reveal the relationship between enzyme activity and the mutation, we performed genetic analysis. Direct sequencing of HEXB exons revealed the presence of two mutations in the patient: c.1021delA in exon 8; and c.1514G $>$ A in exon 13 (Figure 3). Genetic analysis of the patient's family members showed that the mother was heterozygous for c.1021delA, and that the father was heterozygous for c.1514G $>$ A.

The c.1021delA mutation may cause a frameshift at amino-acid position 341 and create a translational stop at amino-acid position 371 in the HEXB protein (p.Ser341ValfsX30). The protein is expected to be truncated at $\sim 60 \%$ of its full length. The nucleotide change of c.1514G $>$ A produces the substitution p.Arg505Gln. To ascertain whether this was not a single-nucleotide polymorphism, we performed direct sequencing of this region in 103 healthy controls, and found that none of them carried this alternative allele. The $\beta$-Hex A with p.Arg505Gln was shown to become a heat-labile enzyme, in previous reports. ${ }^{18,28,29}$ The amino acid at position p.Arg505 is not located in the catalytic site or in the interface between subunits. However, according to Maier et al., ${ }^{21}$ p.Arg505 seems important for dimerization of subunits. A short hydrogen bond between p.Arg505 and Arg501 may be associated with dimerization. The substitution p.Arg505Gln may disrupt this hydrogen bond, which would affect the dimerization of subunits.

\section{Western blot analysis}

To investigate the mutation effect of p.Ser341ValfsX30 and p.Arg505Gln on expression levels of $\alpha$ - and $\beta$-subunit proteins, we performed western blot analysis using total protein in peripheral leukocytes. The control was a healthy volunteer who was confirmed as having no mutations in HEXB. $\beta$-Hex A and total $\beta$-Hex activities of the control were 510.1 and $1539.7 \mathrm{nmol}$ per mg protein per hour, respectively. The molecular weights of subunits were detected (as described below) and were consistent with a previous report. ${ }^{30}$ The precursor and mature forms of the $\alpha$-subunits ( $\alpha p$ and $\alpha \mathrm{m}$ ) were 67 and $54 \mathrm{kDa}$, respectively (Figure $4 \mathrm{a})$. The precursor of $\beta$-subunit $(\beta \mathrm{p})$ was $63 \mathrm{kDa}$, and the mature forms of the $\beta$-subunits $(\beta \mathrm{m})$ were 52 and $29 \mathrm{kDa}$ (Figure 4c). To produce the mature form, the precursors of the $\beta$-subunits were divided into two peptides through proteolytic processing in the lysosome. The band at $52 \mathrm{kDa}$ corresponds to the complex of two peptides, and that at $29 \mathrm{kDa}$ corresponds to the separated peptide. An anti- $\beta$-subunit antibody that we used recognized the N-terminal peptide. The electrophoretic density of each subunit was analyzed using ImageJ (http://rsbweb.nih.gov/ij/), and corrected to its respective density of tubulin. The relative amounts (electrophoretic densities) of the each subunit compared with the control are shown in Figures $4 \mathrm{~b}$ and $\mathrm{d}$ (bar chart). The amount of $\beta \mathrm{m}$ is shown as the sum of bands in 52 and $29 \mathrm{kDa}$ (Figure $4 \mathrm{~d}$ ).

The amount of $\beta$-subunits of the father having p.Arg505Gln was sufficient, even compared with normal subjects, whereas the amount a

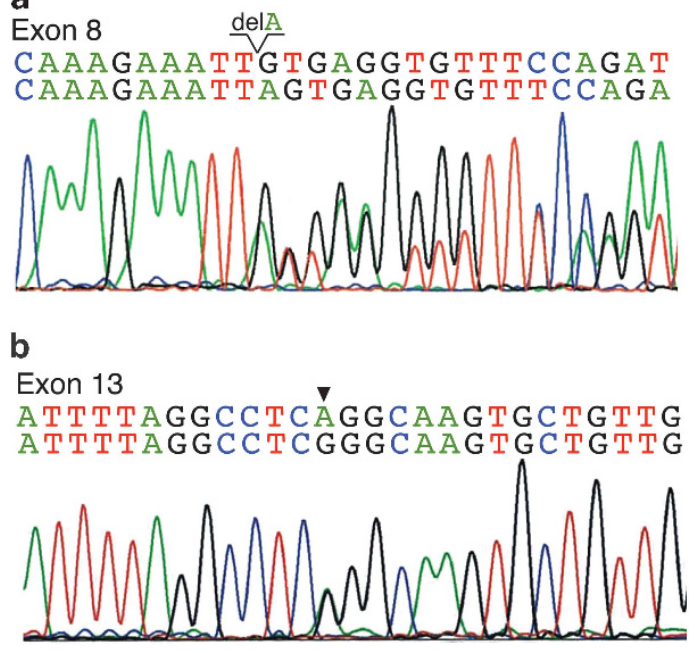

Figure 3 Genetic analysis of HEXB. (a) Heterozygous mutation, c.1021delA (Ser341ValfsX30). (b) Heterozygous mutation, c.1514G >A (Arg505GIn).

of $\beta$-subunits of the mother having p.Ser341ValfsX30 reduced to less than half the amount of the father or the control (Figure 4d). From this result, it was inferred that the translated product from the mutant allele of p.Ser341ValfsX30 could not become the $\beta$-subunit because of a totally different form and/or degradation in the endoplasmic reticulum. The amount of $\beta$-subunits of the patient with p.Ser341ValfsX30 and p.Arg505Gln was reduced further than that of the mother (Figure 4d). It seems that with the addition of the mutation effect of p.Arg505Gln to the reduction amount of $\beta$-subunits, the amount of $\beta$-subunits was further decreased. It was observed that, as with the $\beta$-subunits, the amounts of mature form of the $\alpha$-subunits of the patient decreased markedly compared with those of the mother, father and control (Figure $4 \mathrm{~b}$ ). It is thought that p.Arg505Gln can affect the maturation of $\alpha$-subunits.

\section{Molecular simulation analysis}

To elucidate the pathological significance of p.Arg505Gln, especially the conformational stability of the $\alpha \beta$-heterodimer and the catalytic ability of $\beta$-Hex A in lysosomes, we performed molecular simulation analysis with the mutant $\beta$-subunit. Docking simulation of the $\alpha$-and $\beta$-subunits was carried out using ZDOCK software, which yielded a ZDOCK score that indicated conformational stability. The ZDOCK scores of wild-type $\beta$-Hex A (the wild-type $\alpha$ - and $\beta$-subunits) and mutant $\beta$-Hex A (the wild-type $\alpha$-subunit and mutant $\beta$-subunit) (mean \pm s.d.) were $230.07 \pm 20.77$ and $168.20 \pm 11.49$, respectively. The ZDOCK scores for mutant $\beta$-Hex A were significantly lower than those of wild-type $\beta$-Hex A $(P<0.001)$. This indicated that mutant $\beta$-Hex A decreased in stability compared with wild-type $\beta$-Hex A.

In wild-type $\beta$-Hex $\mathrm{A}$, the guanidyl $\mathrm{N \eta H1}$ of p.Arg505 forms two hydrogen bonds to the backbone oxygen atom or p.Arg501, as described by Maier et al. In our simulation, these hydrogen bonds disappeared in mutant $\beta$-Hex A (the wild-type $\alpha$-subunit and mutant $\beta$-subunit) (Figure 5a). Surface potential analysis using Adaptive Poisson-Boltzmann Solver (APBS) software (http://www.poissonboltzmann.org/apbs/) showed that, in mutant $\beta$-Hex $A$, a slight change in surface potential occurred in the docking sites of the $\alpha$ - and $\beta$-subunits (Figure $5 \mathrm{~b}$ ). Therefore, the change of hydrogen bonds and surface potentials in mutant $\beta$-Hex A mentioned above may affect mutant $\alpha \beta$-heterodimer formation. 

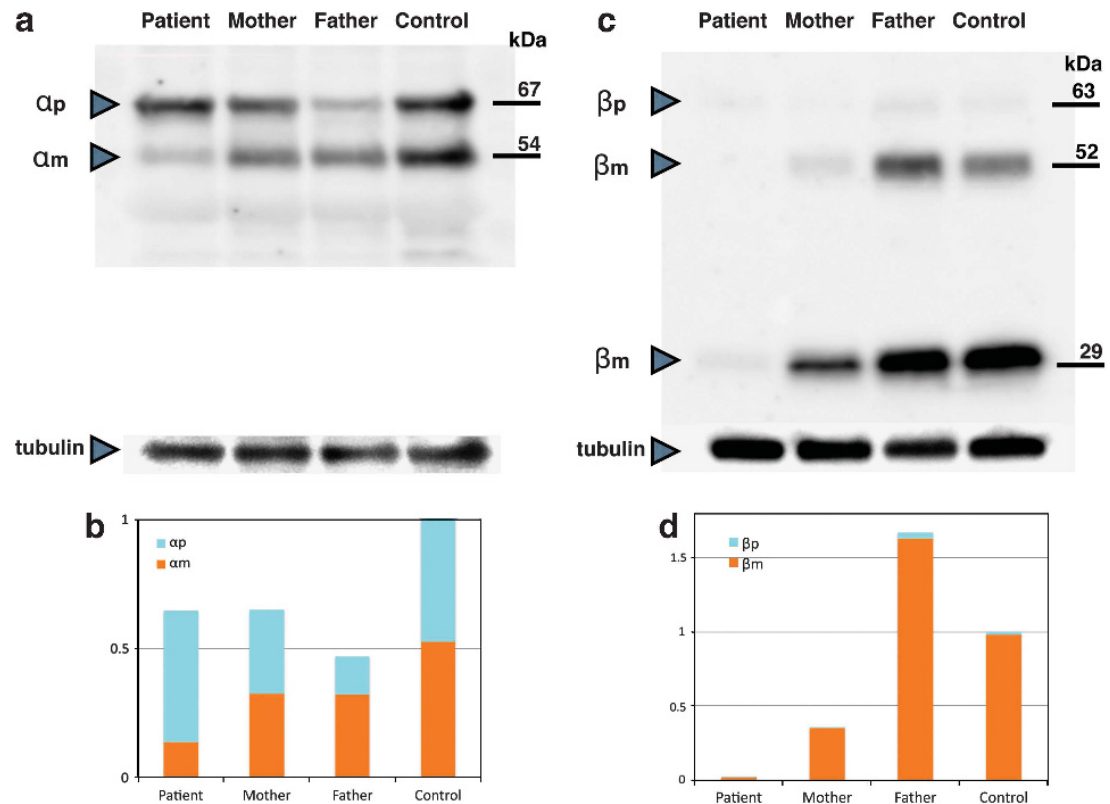

Figure 4 Western blot analysis of $\alpha$ - and $\beta$-subunits. (a, c) Western blot analysis using anti- $\alpha$-subunit and anti- $\beta$-subunit, respectively. (b, d) The relative amount of $\alpha$ - and $\beta$-subunits, respectively, of the patient and the patient's parents, based on the amount of $\alpha$ - and $\beta$-subunits of the control. The precursors of the $\alpha$ - and $\beta$-subunits are denoted as $\alpha \mathrm{p}$ and $\beta \mathrm{p}$, respectively. The mature forms of the $\alpha$ - and $\beta$-subunits are denoted as $\alpha \mathrm{m}$ and $\beta \mathrm{m}$, respectively.
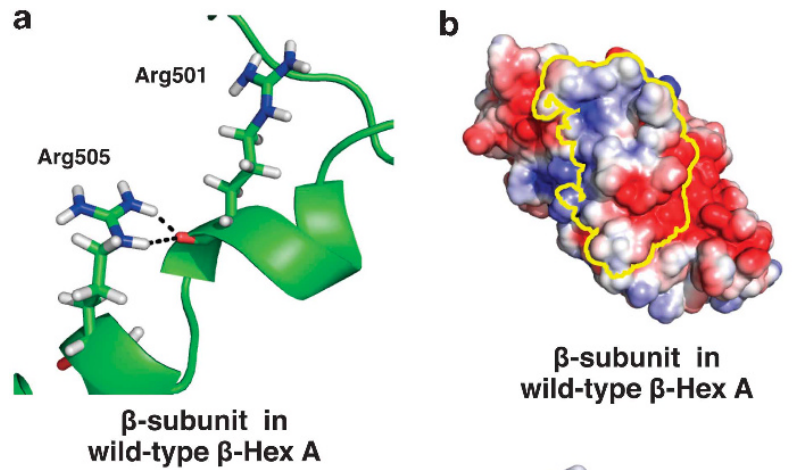

$\beta$-subunit in wild-type $\beta$-Hex A
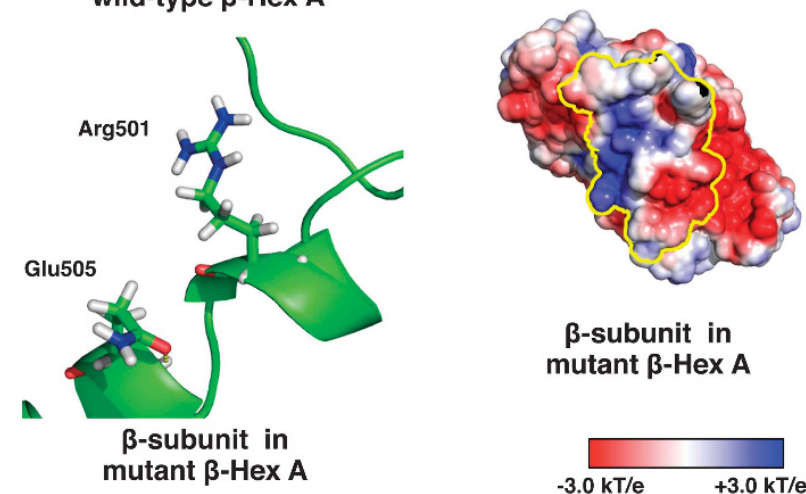

B-subunit in mutant $\beta$-Hex A

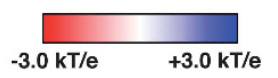

Figure 5 Structural comparison between wild-type $\beta$-Hex $A$ and mutant $\beta$-Hex A. (a) Hydrogen bonds between Arg501 and Arg505 in the $\beta$-subunit. In wild-type $\beta$-Hex $A$, the guanidyl $\mathrm{N} \eta \mathrm{H} 1$ of Arg505 forms two hydrogen bonds to the backbone oxygen atom or Arg501 (black dotted line). The hydrogen bonds disappeared in mutant $\beta$-Hex A. (b) Electrostatic surface potentials of the binding site of $\alpha$ - and $\beta$-subunits. Surface potentials of $\beta$-subunit are shown in PyMOL using APBS. The area surrounded by the yellow line is the docking site of the $\alpha$ - and $\beta$-subunits. In mutant $\beta$-Hex A, a slight potential change in the docking site occurred.
In docking simulations of the GM2AP/GM2 complex and $\beta$-Hex A, the 3-D structure of the GM2AP/GM2/ $\beta$-Hex A complex that resulted was superimposed onto a structure constructed according to the model predicted by Mark et al., ${ }^{19}$ and the number of structures with an RMSD of $\leqslant 5 \AA$ were counted. The number of structures with an RMSD of $\leqslant 5 \AA$ per 45000 runs for wild-type and mutant $\beta$-Hex A (mean \pm s.d.) were $14.00 \pm 8.00$ and $9.17 \pm 6.85$, respectively. The number of mutant $\beta$-Hex A was significantly lower than that of wild-type $\beta$-Hex A $(P<0.01)$. GM2AP transports GM2 and presents it to the active site of the $\alpha$-subunit. ${ }^{2,19}$ This result indicated that the GM2AP/GM2 binding abilities of mutant $\beta$-Hex A were lower than those of wild-type $\beta$-Hex A, and mutant $\beta$-Hex A may reduce the chance of proper substrate presentation from GM2AP.

In the docking simulations of GM2 and $\beta$-Hex $A$, the number of structures that met the following conditions was counted: (1) the distance of $\leqslant 5 \AA$ between the oxygen atom in the glycosidic bond of the GM2 terminal $\beta$-linked GalNac and the oxygen atom of the carboxylate group in Glu323 of $\alpha$-subunit; and (2) GM2 oriented so that the C2-acetamido group of the GM2 faced the oxygen atom of the carboxylate group in Asp322 of $\alpha$-subunit. ${ }^{19,20}$ The numbers of structures with the above conditions per 45000 runs for wild-type and mutant $\beta$-Hex A (mean \pm s.d.) were $10708.17 \pm 97.17$ and $9565.00 \pm 44.43$, respectively. The number of mutant $\beta$-Hex A was significantly lower than that of wild-type $\beta$-Hex A $(P<0.001)$. This indicated that mutant $\beta$-Hex A has less favorable orientation of GM2 and reduced GM2 hydrolysis ability.

\section{DISCUSSION}

Here, we present the result from our investigation of a Japanese female with adult SD, with a relatively early disease onset. The patient developed symptoms at 6 years of age. At her first clinical examination, when she was 20 years old, she presented ataxia in her trunk and limbs, muscle atrophy, and limb weakness. The age of the patient at disease onset was 10-20 years younger than that of a typical patient 
with adult SD. However, our patient's symptoms were comparable to those of adult SD, involving only the cerebellum and lower motor neurons. ${ }^{4,5,10}$ Juvenile SD affects a wider range of nerves. ${ }^{3}$ In addition, the slow disease progression in this patient was consistent with adult SD.

In genetic analysis, the patient had compound heterozygous mutations, c.1021delA (p.Ser341ValfsX30) and c.1514G $>$ A (p.Arg505Gln), in HEXB. The mother of the patient had a frameshift mutation, p.Ser341ValfsX30, which created a translational stop, and her enzyme activity reduced by about half. The enzyme activity of the father, who had p.Arg505Gln, was equivalent to that of the controls. To investigate the pathological significance of p.Arg505Gln and the mechanisms leading to disease onset in the patient, we performed western blot analysis and molecular simulation analysis with the mutant $\beta$-subunit.

From the results of western blot analysis, the $\beta$-subunit was not produced from a mutant allele of p.Ser341ValfsX30. The enzyme activity of $\beta$-Hex A was not decreased to the threshold level of that required for developing $\mathrm{SD}$, from the twofold reduction of the $\beta$ subunit alone. However, with the additional effects of p.Arg505Gln to p.Ser341ValfsX30, the enzyme activity of $\beta$-Hex A was further decreased.

One of the effects of p.Arg505Gln was thought to be a hampering of the maturation of $\beta$-Hex $A$, as western blot analysis showed that the amounts of the mature forms of $\alpha$ - and $\beta$-subunits were markedly lower in the patient. p.Arg505Gln may have an influence on the dimerization of $\alpha \beta$-subunits from its location, and our docking simulation showed the disappearance of two hydrogen bonds between p.Arg505 and p.Arg501 associated with dimerization and change in surface potential in the docking sites of the mutant $\beta$-subunit. Dimerization of the $\alpha$ - and $\beta$-subunits was indispensable for the process of maturation of the enzyme. ${ }^{2,16,19}$ The precursor form of $\beta$-Hex A, although not analyzed in this study, has the same binding sites as the $\alpha$-and $\beta$-subunits of the mature form of $\beta$-Hex A. If a similar conformational change in the mature form of $\beta$-Hex A occurs in the precursor form, the process of maturation of $\beta$-Hex A may be hampered not only in lysosomes but also in the endoplasmic reticulum and Golgi bodies. ${ }^{2,31}$ Each subunit can take a maturation process in the form of $\beta \beta$-homodimer and $\alpha \alpha$ homodimer also. ${ }^{2}$ The difference in stability of each dimer was reported, in the order of $\beta \beta>\alpha \beta>>\alpha \alpha^{2}$ If the stability would change to $\beta \beta>>\alpha \beta$ owing to the p.Arg505Gln mutation, although not analyzed in this study, much more mature $\beta$-subunits might be derived from the $\beta \beta$-homodimer, and the amount of the mature $\alpha$-subunit might be decreased, as seen in the result of western blot analysis of the father (Figure 4d).

Another effect of p.Arg505Gln was thought to be a hindering of the catalytic ability of $\beta$-Hex A. Our docking simulation of the $\alpha$ - and $\beta$-subunits showed that p.Arg505Gln mutation reduced the conformational stability of mutant $\beta$-Hex A, which may alter the binding site of GM2AP/GM2 or GM2. ${ }^{21}$ This hypothesis was supported by the significantly lower numbers of properly binding GM2AP/GM2 and GM2 to mutant $\beta$-Hex A observed in our docking simulation analysis. Each step in GM2AP/GM2/ $\beta$-Hex A complex formation was impaired by the mutant $\beta$-subunit, which may have resulted in a synergistic negative effect on $\beta$-Hex A activity.

Although p.Arg505Gln is predicted to have a negative effect on $\beta$ Hex A activity, SD patients homozygous for p.Arg505Gln show a late disease onset and very mild symptoms according to a report by Delnooz et al., ${ }^{7}$ indicating that a p.Arg505Gln mutation has a limited negative effect on $\beta$-Hex A activity; the activity of $\beta$-Hex A with a mutated $\beta$-subunit protein with p.Arg505Gln is maintained to some extent.

Our patient, who carried a p.Arg505Gln mutation and a frameshift mutation in HEXB, showed earlier onset and more severe symptoms than those seen in patients homozygous for p.Arg505Gln. The clinical discrepancy between our patient and patients homozygous for p.Arg505Gln may be due to expression levels of $\beta$-subunit protein.

There are two possible treatment strategies for our patient. One is to enhance the expression levels of mutant $\beta$-subunit protein with p.Arg505Gln. Transcription-activating drugs, such as histone deacetylase inhibitors, may increase the expression levels of mutant $\beta$-subunit protein and ameliorate the severity of the clinical symptoms. The other treatment is chaperone therapy, which increases the conformational stability of $\beta$-Hex A. ${ }^{31}$ The chemical compounds working as chaperones may also improve residual $\beta$-HexA activity.

In conclusion, various methods, including simulation analysis, are useful aids in understanding the effects of the p.Arg505Gln mutation on molecular conformation, and insights into the molecular pathology are helpful in developing novel treatments for lysosomal disorders.

\section{ACKNOWLEDGEMENTS}

We greatly appreciate the technical assistance provided by Yuko Ando. We also thank Kobayashi Kazuhiro, Kanagawa Motoi, Satake Wataru and Takehiro Ueda for their helpful suggestions.

1 Gravel, R., Kaback, M. M. \& Proia, R. L. The GM2 gangliosidoses. The Metabolic and Molecular Basis of Inherited Disease (eds Scriver, C. R., Beaudet, A. L., Sly, W. S., Valle, D., Childs, B. \& Vogelstein, B.) 3827-3876 (McGraw-Hill, Inc., New York, NY USA, 2001).

2 Mahuran, D. J. Biochemical consequences of mutations causing the GM2 gangliosidoses. Biochim. Biophys. Acta. 1455, 105-138 (1999).

3 Maegawa, G. H., Stockley, T., Tropak, M., Banwell, B., Blaser, S., Kok, F. et al. The natural history of juvenile or subacute GM2 gangliosidosis: 21 new cases and literature review of 134 previously reported. Pediatrics 118, e1550-e1562 (2006).

4 Redonnet-Vernhet, I., Mahuran, D. J., Salvayre, R., Dubas, F. \& Levade, T. Significance of two point mutations present in each HEXB allele of patients with adult GM2 gangliosidosis (Sandhoff disease) homozygosity for the Ile207->Val substitution is not associated with a clinical or biochemical phenotype. Biochim. Biophys. Acta. 1317, 127-133 (1996)

5 Hara, A., Uyama, E., Uchino, M., Shimmoto, M., Utsumi, K., Itoh, K. et al. Adult Sandhoff's disease: R505Q and I207V substitutions in the HEXB gene of the first Japanese case. J. Neurol. Sci. 155, 86-91 (1998).

6 Oonk, J. G., van der Helm, H. J. \& Martin, J. J. Spinocerebellar degeneration: hexosaminidase A and B deficiency in two adult sisters. Neurology 29, 380-384 (1979)

7 Delnooz, C. C., Lefeber, D. J., Langemeijer, S. M., Hoffjan, S., Dekomien, G., Zwarts, M. J. et al. New cases of adult-onset Sandhoff disease with a cerebellar or lower motor neuron phenotype. J. Neurol. Neurosurg. Psychiatry. 81, 968-972 (2010).

8 Yoshizawa, T., Kohno, Y., Nissato, S. \& Shoji, S. Compound heterozygosity with two novel mutations in the HEXB gene produces adult Sandhoff disease presenting as a motor neuron disease phenotype. J. Neurol. Sci. 195, 129-138 (2002).

9 Hancock, L. W., Horwitz, A. L., Cashman, N. R., Antel, J. P. \& Dawson, G. N-acetylbeta-hexosaminidase $B$ deficiency in cultured fibroblasts from a patient with progressive motor neuron disease. Biochem. Biophys. Res. Commun. 130, 1185-1192 (1985)

10 Takado, Y., Koide, T., Yoshikawa, K., Amaya, N., Yoshida, Y. \& Ishiguro, H. A patient with GM2 gangliosidosis presenting with motor neuron disease symptom in his forties. Rinsho. Shinkeigaku. 47, 37-41 (2007)

11 Schnorf, H., Gitzelmann, R., Bosshard, N. U., Spycher, M. \& Waespe, W. Early and severe sensory loss in three adult siblings with hexosaminidase A and B deficiency (Sandhoff disease). J. Neurol. Neurosurg. Psychiatry. 59, 520-523 (1995).

12 Hou, Y., Tse, R. \& Mahuran, D. J. Direct determination of the substrate specificity of the alpha-active site in heterodimeric beta-hexosaminidase A. Biochemistry 35 3963-3969 (1996).

13 Suzuki, K. Enzymatic diagnosis of sphingolipidoses. Methods. Enzymol. 138, 727-762 (1987)

14 Sandhoff, K., Harzer, K. \& Fürst, W. Sphingolipid activator proteins. The Metabolic and Molecular Basis of Inherited Disease (eds Scriver, C. R., Beaudet, A. L., Sly, W. S. \& Valle, D.) 2427-2441 (McGraw-Hill, Inc., New York, NY, USA, 1995). 
15 Tropak, M. B. \& Mahuran, D. Lending a helping hand, screening chemical libraries for compounds that enhance beta-hexosaminidase A activity in GM2 gangliosidosis cells. Febs. J. 274, 4951-4961 (2007).

16 Proia, R. L., d'Azzo, A. \& Neufeld, E. F. Association of alpha- and beta-subunits during the biosynthesis of beta-hexosaminidase in cultured human fibroblasts. J. Biol. Chem. 259, 3350-3354 (1984).

17 Lowry, O. H., Rosebrough, N. J., Farr, A. L. \& Randall, R. J. Protein measurement with the Folin phenol reagent. J. Biol. Chem. 193, 265-275 (1951).

18 Narkis, G., Adam, A., Jaber, L., Pennybacker, M., Proia, R. L. \& Navon, R. Molecular basis of heat labile hexosaminidase B among Jews and Arabs. Hum. Mutat. 10, 424-429 (1997).

19 Mark, B. L., Mahuran, D. J., Cherney, M. M., Zhao, D., Knapp, S. \& James, M. N. G. Crystal structure of human $\beta$-hexosaminidase $b$ : understanding the molecular basis of Sandhoff and Tay-sachs disease. J. Mol. Biol. 327, 1093-1109 (2003).

20 Lemieux, M. J., Mark, B. L., Cherney, M. M., Withers, S. G., Mahuran, D. J. \& James, M. N. Crystallographic structure of human beta-hexosaminidase A: interpretation of Tay-Sachs mutations and loss of GM2 ganglioside hydrolysis. J. Mol. Biol. 359, 913-929 (2006).

21 Maier, T., Strater, N., Schuette, C., Klingenstein, R., Sandhoff, K. \& Saenger, W. The X-ray crystal structure of human $\beta$-hexosaminidase $B$ provides new insights into Sandhoff disease. J. Mol. Biol. 328, 669-681 (2003).

22 Desriani, Ferri S. \& Sode, K. Amino acid substitution at the substrate-binding subsite alters the specificity of the Phanerochaete chrysosporium cellobiose dehydrogenase. Biochem. Biophys. Res. Commun. 391, 1246-1250 (2010).
23 Wang, M. \& Wang, J. A computerized protein-protein interaction modeling study of ampicillin antibody specificity in relation to biosensor development. J. Mol. Model. 17, 2873-2882 (2011).

24 Chen, R., Li, L. \& Weng, Z. ZDOCK: an initial-stage protein-docking algorithm. Proteins 52, 80-87 (2003).

25 Okai, M., Kubota, K., Fukuda, M., Nagata, Y., Nagata, K. \& Tanokura, M. Crystal structure of gamma-hexachlorocyclohexane Dehydrochlorinase LinA from Sphingobium japonicum UT26. J. Mol. Biol. 403, 260-269 (2010).

26 Wright, C. S., Zhao, Q. \& Rastinejad, F. Structural analysis of lipid complexes of GM2activator protein. J. Mol. Biol. 331, 951-964 (2003).

27 Ihaka, R. \& Gentleman, R. R: A language for data analysis and graphics. J. Comp. Graph Stat. 5, 299-314 (1996).

28 Bolhuis, P. A., Oonk, J. G., Kamp, P. E., Ris, A. J., Michalski, J. C., Overdijk, B. et al. Ganglioside storage, hexosaminidase lability, and urinary oligosaccharides in adult Sandhoff's disease. Neurology 37, 75-81 (1987).

29 Bolhuis, P. A., Ponne, N. J., Bikker, H., Baas, F. \& Vianney de Jong, J. M. Molecular basis of an adult form of Sandhoff disease: substitution of glutamine for arginine at position 505 of the beta-chain of beta-hexosaminidase results in a labile enzyme. Biochim. Biophys. Acta. 1182, 142-146 (1993).

30 Hasilik, A. \& Neufeld, E. F. Biosynthesis of lysosomal enzymes in fibroblasts. synthesis as precursors of higher molecular weight. J. Biol. Chem. 255, 4937-4945 (1980).

31 Maegawa, G. H., Tropak, M., Buttner, J., Stockley, T., Kok, F., Clarke, J. T. et al. Pyrimethamine as a potential pharmacological chaperone for late-onset forms of GM2 gangliosidosis. J. Biol. Chem. 282, 9150-9161 (2007).

Supplementary Information accompanies the paper on Journal of Human Genetics website (http://www.nature.com/jhg) 